\title{
Low Cost Sensor Based Obstacle Detection and Description
}

\section{Experiments with Mobile Robots using Grid Representation*}

\author{
Paulo Menezes, Jorge Dias, Helder Araújo, Aníbal de Almeida \\ Instituto de Sistemas e Robótica \\ Departamento de Engenharia Electrotécnica \\ Largo Marquês de Pombal, 3000 COIMBRA, PORTUGAL \\ \{paulo,jorge,helder,adealmeida\}@isr.uc.pt
}

\begin{abstract}
This article describes an algorithm for detection of obstacles, through the use of low cost sonar sensors, for the navigation of mobile robots. The use of a grid representation for the cartesian space, associated to probabilistic operations on it, allowed the implementation of a fast, simple and robust algorithm for obstacle detection. This algorithm runs always that the mobile robot navigates, and allows to overcome obstacles that appear unexpectedly in the robot's path. The information used to represent the obstacles is based in the information provided by sonar sensors. A stochastic model for the sonar sensors and their activity, associated to the platform motion, are explored by the algorithm. Some experimental results obtained through the use this algorithm are described at the end of the article.
\end{abstract}

\section{Introduction}

The operation of a mobile robot in a unstructured environment is only possible, if the robot has ability to cope with changes in the environment. These changes, which include the presence of new obstacles, can be detected using sensorial systems.

In most cases a trajectory planner, using some a priori description of the robot's workspace, generates feasible paths to execute the desired missions. Using sensorial information, it is possible to improve the mobile robot capabilities, allowing a realtime reaction to avoid the collision and/or to re-plan the trajectory as proposed by Chatila [2].

Normally sonar sensors are chosen to acquire information around the mobile robot. Although sonar sensors present several problems which are related with the limited information that can be obtained from raw data. The choice was based mainly on their low cost, the ease of their use and the relatively low computational power required.

*This research was partially sponsored by NATO's Scientific Affairs Division in the framework of the Science for Stability Program 


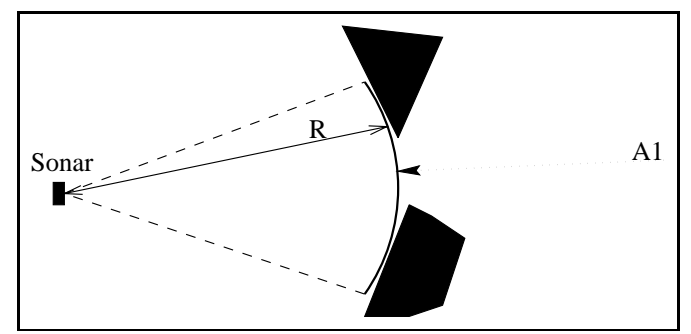

Figure 1. Both targets $\mathrm{T} 1$ and $\mathrm{T} 2$ produce the same range reading.

\section{Problem formulation}

The aim of this work is to allow the collision free navigation of a mobile robot in a semi-structured environment through the use of sensorial information. The inputs available are the range data provided by the ultrasonic range sensors, the robot's position and orientation, and the direction of motion. Range data and the localization of the robot are used to detect the presence of obstacles and their possible localization.

The direction of motion is used to explore the sensors' activity. Robot's activity serves two purposes: the first is to determine which region is of concern, because we don't need to care about obstacles located in regions where we are not going through, and using this information we can decide which set of sensors should be scanned. This has implication on the time processing since scanning more sensors is time costly both in data acquisition and also in data processing. The second reason is that, for a given location, multiple readings performed by a static sensor don't bring any new information about the extent of the obstacle, although they could be averaged to remove sensor noise.

\subsection{Uncertainty in sonar range measures}

In the majority of the mobile robots that use sonar sensors, the range information is obtained from the time-of-flight (t.o.f.). However from these measures, the only information that can be obtained is the distance to the closest point of the obstacle that reflected the wave, back to the sensor. This is not sufficient to characterize the obstacle, whose presence was detected. The wide opening angle presented by most sonar sensors introduces another uncertainty factor: to which direction corresponds the measure? Figure 1 illustrates a situation where two possible obstacles could produce the same range reading. From a range value we can only say that there is a region in which every point is a possible location for the detected obstacle. Considering the cartesian space and ignoring some errors that appear in range values, this region takes the form of one arc centered in the sensor, with radius equal to the range reading and angular value equal to twice the sensor opening.

It is also clear that any target tangent to the arc $A 1$ would produce the same result. By this reason this arc is commonly referred as a Region of Constant Depth (RCD)[3], which is nothing more than a set of points equally candidates to be considered as the one that has produced the echo.

Calibration experiments performed in our lab have shown that sonar sensors can be approximated as ideal sensors whose outputs are corrupted with gaussian noise of zero mean and standard deviation given by the following expression 


$$
\sigma_{D}(z)=0.0052 \times z+0.002[m]
$$

where $z$ is the distance between the sensor and the target in meters [5].

Using this model we can write the following expression that gives the probability of obtaining a sonar reading $r$ given a target at a distance $z$.

$$
p(r \mid z)=\frac{1}{\sqrt{2 \pi \sigma_{D}}} \exp \left(\frac{-(r-z)^{2}}{2 \sigma_{D}^{2}}\right)
$$

\subsection{Representation}

As we have shown above, to obtain a description of the obstacles in terms of extents and orientation, these have to be observed from multiple points of view, due to the poor information that can be extracted from each observation. Here arises a problem, since our main goal is navigation we can not move the robot around an obstacle to obtain its full description. We, as long as possible, have to acquire information while the robot is moving. But to do so a suitable representation has to be used, allowing the fusion of information obtained in different robot's positions. The choice was to use a discretization of the operational space by dividing it into cells, each one representing a small area. For each cell, a value is assigned that represents the knowledge level regarding its occupancy. This value is updated, each time the area it represents, is scanned. In our implementation, a cell size of $10 \times 10 \mathrm{~cm}$ was used as a compromise between the smallest detectable object and computational load. Notice that decreasing these dimensions has direct implications on the amount of information that must be processed and is represented by this grid structure. Algorithms operating on these type of structures have a general trend for $O\left(n^{2}\right)$ computational complexity and consequently in the increase of the processing time.

\subsection{Obstacle Detection}

Using the stochastic sensor model shown in equation (2), and using Bayes' formula we can obtain an expression for the existence of an obstacle $(O)$, given a range measure, as suggested by Elfes [4]

$$
p(O \mid r)=\frac{p(r \mid o) p(o)}{p(r \mid o) p(o)+p(r \mid u) p(u)}
$$

In this equation the letter $o$ means occupied and $u$ means unoccupied and could be used to estimate each cell state.

Since the RCD length grows with distance and, with it the uncertainty about the target location, only range measures below 3 meters are used. This has the advantage of reducing the errors produced by multiple reflections and also reduce processing time, because the number of cells that have to be updated is smaller.

Substituting these values in expression (1), we can see that the standard deviation of range errors are $\sim 6$ times smaller than the cell size we chose. From this it can be seen that the sensor model can be substituted by a step model like the one shown in figure 2 in order to reduce computation time.

So, for a range value $r$, there is a set of cells that enclose all possible target locations that could produce this value. This set also contains the arc above referred to as RCD (figure 3 ) and can be viewed as its discretization.

For short range values, the RCD is contained on a single cell, the meaning being

that it contains surely the point that produced the echo. In these cases it is assigned 


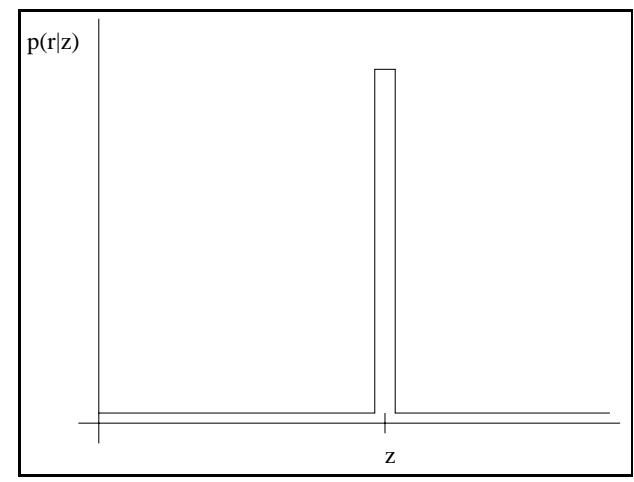

Figure 2. Approximation of the sonar measures by a step model

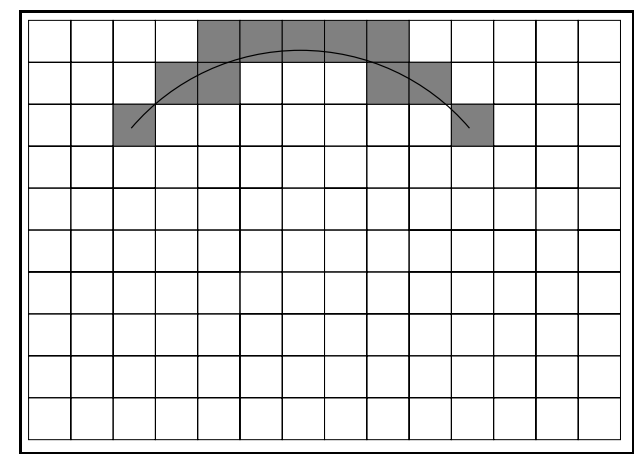

Figure 3. A discrete RCD

a probability of $100 \%$ of occupancy. For larger range values the RCD spans over several cells, and then this certainty must be shared by all of them. So each one is updated by $1 / n$, where $\mathrm{n}$ is the number of cells that contain the RCD.

\subsection{Updating the grid}

Once a sensor measurement is obtained, several steps are needed; obtaining the robot's position and orientation at the point where the measurement was taken, the determination of the sensor world coordinates is given by:

$$
{ }^{W} \overrightarrow{\mathbf{x}}={ }^{W} \mathbf{T}_{S_{i}} *{ }^{S_{i}} \overrightarrow{\mathbf{x}}
$$

where ${ }^{W} \overrightarrow{\mathbf{x}}$ are the sensor world coordinates, $\mathbf{T}$ the transformation matrix, and ${ }^{S_{i}} \overrightarrow{\mathbf{x}}$ the sensor coordinates related with the robot frame.

For a sensor with an opening angle $\theta$ and a measurement $r$, the cells that must be updated are those that fall inside the cone defined by the two line segments and the arc, as shown in figure 4 . The cells that contain the RCD will get their value increased, and the ones between the sensor and the RCD get it decreased because if there exists an obstacle the range value would be smaller. The letter $E$ signifies that the probability of occupancy in that cell will be decreased and the opposite will be made for the letter $O$.

\subsection{Exploring Sensor Activity}

The information about the localization and/or dimensions of an obstacle using sonar sensors can not be obtained from a single observation. Even when using multiple 


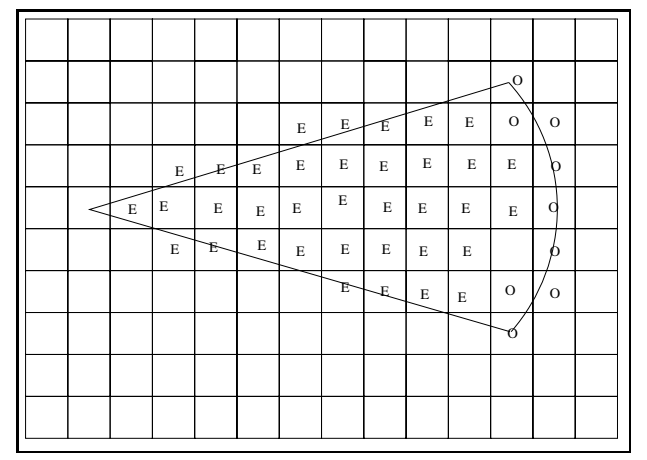

Figure 4. Cells whose probabilities are affected. The letter $E$ signifies that the probability of occupancy in that cell will be decreased. For the letter $O$ the probability will be increased.

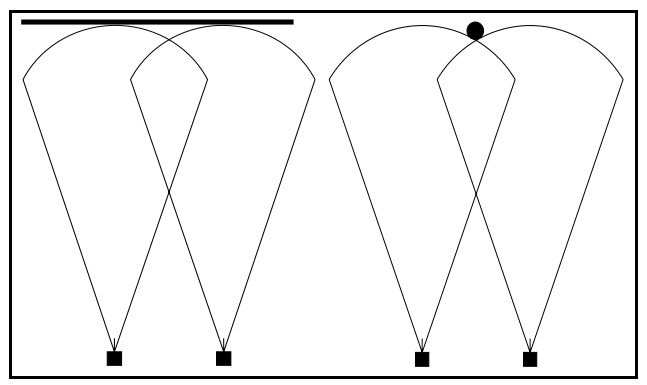

Figure 5. Using two sensors it is not possible to differentiate the kind of target that produced the range values

sensor configurations, the characterization of one obstacle is not possible due to the low spatial sensor resolution.

From figure 5 we can conclude that even using two sensors it is not possible to differentiate between two types of targets because in both situations the range measurements would give the same the values. In fact, although in each scan the grid values are usually slightly changed, after performing a certain number of readings from the same position, all the cells that contain the RCD will reach the maximum level. To overcome this problem, measures are taken only when the robot's position changes.

The information about the direction of motion is also important because, even though the robot has a belt of 24 sensors, only the sensors directed to the regions of interest are scanned allowing once again to reduce the processing time.

\subsection{Potential Grid}

A trajectory finder module was implemented to generate an alternative path after the detection of an obstacle. This module is based in the potential field proposed by Khatib [6]. Potential field based methods have been used to overcome the detected obstacles with success, the only drawback is being that the repulsive forces have to be calculated at every instant. Using the above described approach to detect the obstacles it becomes a time consuming process to calculate the overall repulsive force from the contribution each of the occupied cells. 


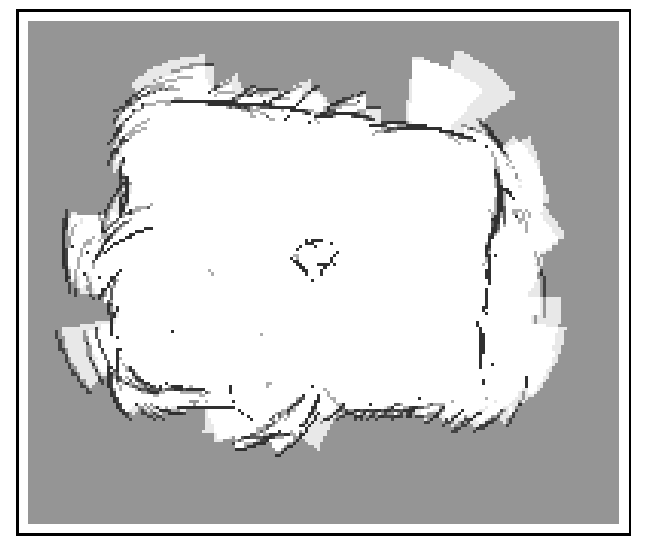

Figure 6. Results obtained in a small room having a vacuum cleaner in the middle

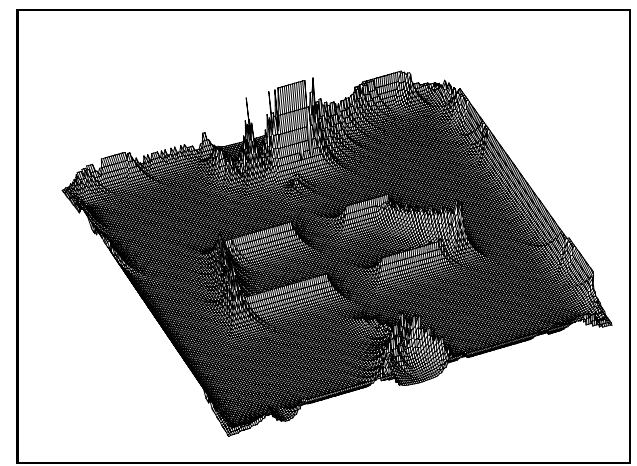

Figure 7. Potential grid generated from simulated sonar scans

However using the measure of the potential field along the path and for each crossed cell, the task for trajectory finder module will be much simpler. This potential grid is constructed from the occupancy grid, by adding to the cell structure another field representing the potential value. This potential grid is calculated from the occupancy grid using the method proposed by Plumer [8], where for each grid cell the corresponding local potential value $\phi_{i j}$ is calculated based on the value of $\phi$ of the 8 neighbor cells.

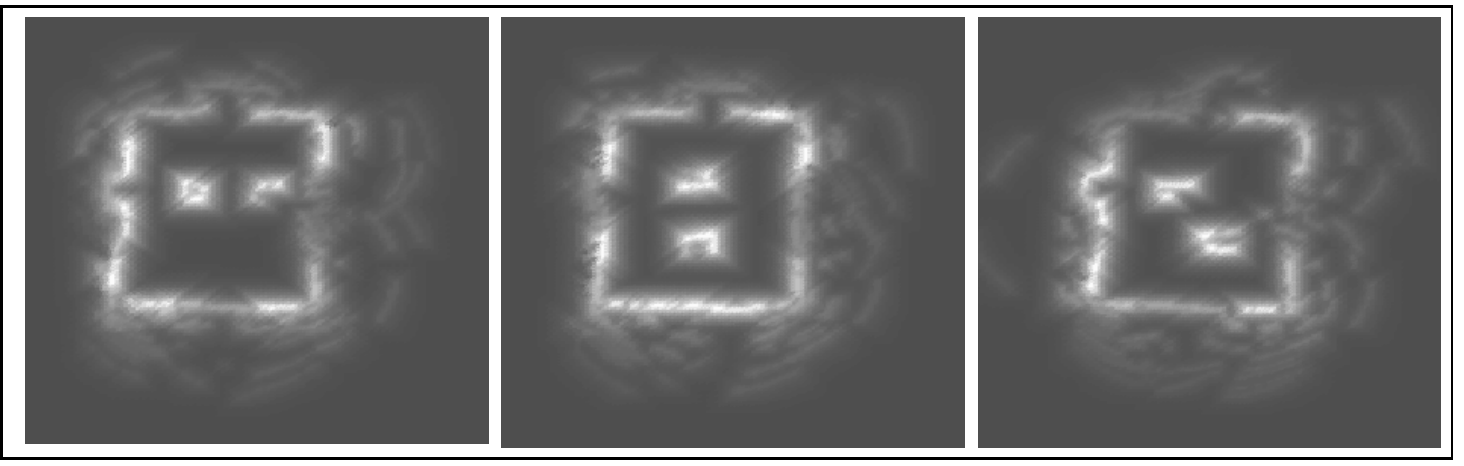

Figure 8. Potential Field obtained for different obstacle configurations 


\section{Results}

Tests have shown good results using the approach described above. Given an a priori map of the laboratory whith some boxes randomly placed in the room, the robot was able to detect their presence and, using the artificial potential field, avoid the collision and reach the goal position. Success was also obtained when using obstacles without planar faces, like baskets and even people.

Figure 6 shows the results of the obstacle detection module after performing some movements in our laboratory, with a vacuum cleaner in the center.

Currently, we are integrating the above described methods to generate the potential grid simultaneously with obstacle detection, in order to allow a faster operation of the robot in the presence of new obstacles. In figure 7 are shown the results obtained simulating the robot and the environment. Figure 8 shows the potential field obtained in a room with three different configurations of the obstacles where dark areas represent low potential values and conversely white areas high potential values. It can be seen that there are marked areas outside the walls, this is due to the occurence of spurious reflections.

\section{Final Remark}

The implemented methods cope with changes in the environment, allowing the operation of a mobile platform in a human environment where the existent objects are subject to change. For example, if an object has been moved, the corresponding cells that were marked as occupied, will be marked as empty and those that correspond to the new position of the box will be marked as occupied. The online generation of a potential grid, allows the faster operation of the obstacle avoidance system.

\section{References}

[1] P. Menezes, H. Araújo, J.Dias, M.I. Ribeiro, "Obstacle Detection in Mobile Robots Using Sonar Data", Associação Portuguesa de Controlo Automático Int. Conf. - Controlo 94, I.S.T., Lisbon, September 14-16, 1994.

[2] R. Chatila, R. Alami, B. Degallaix, H. Laruelle, "Integrated Planning and Execution Control of Autonomous Robot Actions", Proc. IEEE Int. Conference on Robotics \& Automation, Nice, May 1992, pp. 2689-2696.

[3] J.J. Leonard, H.F. Durrant-Whyte, Directed Sonar Sensing for Mobile Robot Navigation, Kluwer Academic Press, 1992.

[4] A. Elfes," Sonar-Based Real-World Mapping and Navigation", IEEE Journal of Robotics and Automation, vol. RA-3, No. 3, June 1987.

[5] F. Moita, A. Feijao, U. Nunes, A. de Almeida, "Modeling and Calibration of an Ultrasonic Ranging System of a Mobile Robot", Associação Portuguesa de Controlo Automático Int. Conf. - Controlo 94, I.S.T., Lisbon, September 14-16, 1994.

[6] O. Khatib, "Real-time obstacle avoidance for manipulators and mobile robots", Proc. IEEE Int. Conference on Robotics \& Automation, 1985, pp. 500-505.

[7] B. Barshan and R. Kuc, "Differentiating Sonar Reflections from Corners and Planes by Employing an Intelligent Sensor", IEEE Trans. on Pattern Analysis and Machine Intelligence, Vol. 12 No. 6 June 1990, pp. 560-569.

[8] E. Plumer, "Neural Network Structure for Navigation Using Potential Fields",IEEE 1992. 